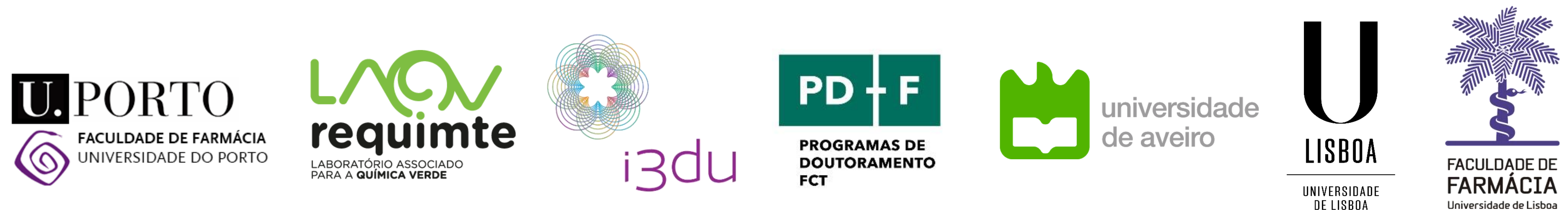

\section{Insight into the protein tyrosine phosphatase 1B (PTP1B) inhibitory activity of pyrazoles}

\section{Sónia Rocha ${ }^{1}$, Daniela Ribeiro ${ }^{1}$, Vera L. M. Silva², Pedro M. O. Gomes ${ }^{2}$, Artur M. S. Silva ${ }^{2}$, Alberto N. Araújo ${ }^{1}$, M. Luísa Corvo ${ }^{3}$, Eduarda Fernandes ${ }^{1}$, Marisa Freitas ${ }^{1}$}

${ }^{1}$ LAQV, REQUIMTE, Laboratory of Applied Chemistry, Department of Chemical Sciences, Faculty of Pharmacy, University of Porto, 4050-313 Porto, Portugal.

2LAQV-REQUIMTE, Department of Chemistry, University of Aveiro, Aveiro, Portugal.

${ }^{3}$ Research Institute for Medicines, Faculty of Pharmacy, University of Lisbon, 1649-003 Lisbon, Portugal.

\section{Introduction}

Diabetes mellitus (DM) is a long-term chronic condition characterized by hyperglycemia, being one of the biggest global health emergencies of the $21^{\text {st }}$ century. Type 2 DM is the most common form of DM, representing 90-95\% of patients with DM. Insulin resistance is the earliest detectable abnormality and the characteristic feature in individuals with type 2 DM. Protein tyrosine phosphatase 1B (PTP1B) has been emerging as a promising drug target for the management of type $2 \mathrm{DM}$. Different causes disrupt a complex network of signaling cascades where insulin is involved, impairing its play role on glucose homeostasis. The insulin binding to the $\alpha$ subunit of its extracellular receptor (IR) (Fig. 1) unleashes the phosphorylation of the tyrosine residues of the $\beta$ subunits, subsequent protein-protein interactions and the phosphorylation of insulin receptor substrates (IRS). Then, IRS proteins serve as docking for other signaling molecules, to onset protein kinase B (PKB) (also known as Akt) activation and translocation of glucose transporter 4 (GLUT4) storage vesicles to the plasma membrane for glucose uptake. PTP1B, as a negative regulator of insulin signaling pathway, acts by dephosphorylation of IR and IRS, leading to the attenuation of the insulin signal. PTP1B inhibitors amplify the level of phosphorylation of the IR and its substrates, rising the translocation of glucose transporters and glucose uptake in insulin-dependent cells, improving insulin sensitivity.

Main goal: Compare the inhibitory effect of a panel of new structurally related pyrazoles (Table 1) against human PTP1B activity, using a microanalysis screening system, and determine the mechanism of inhibition of the most active compounds, graphically by Lineweaver-Burk plots and by non-linear least squares regression using the Solver ${ }^{\mathrm{TM}}$ supplement of Excel Microsoft Office ${ }^{\mathrm{TM}}$.

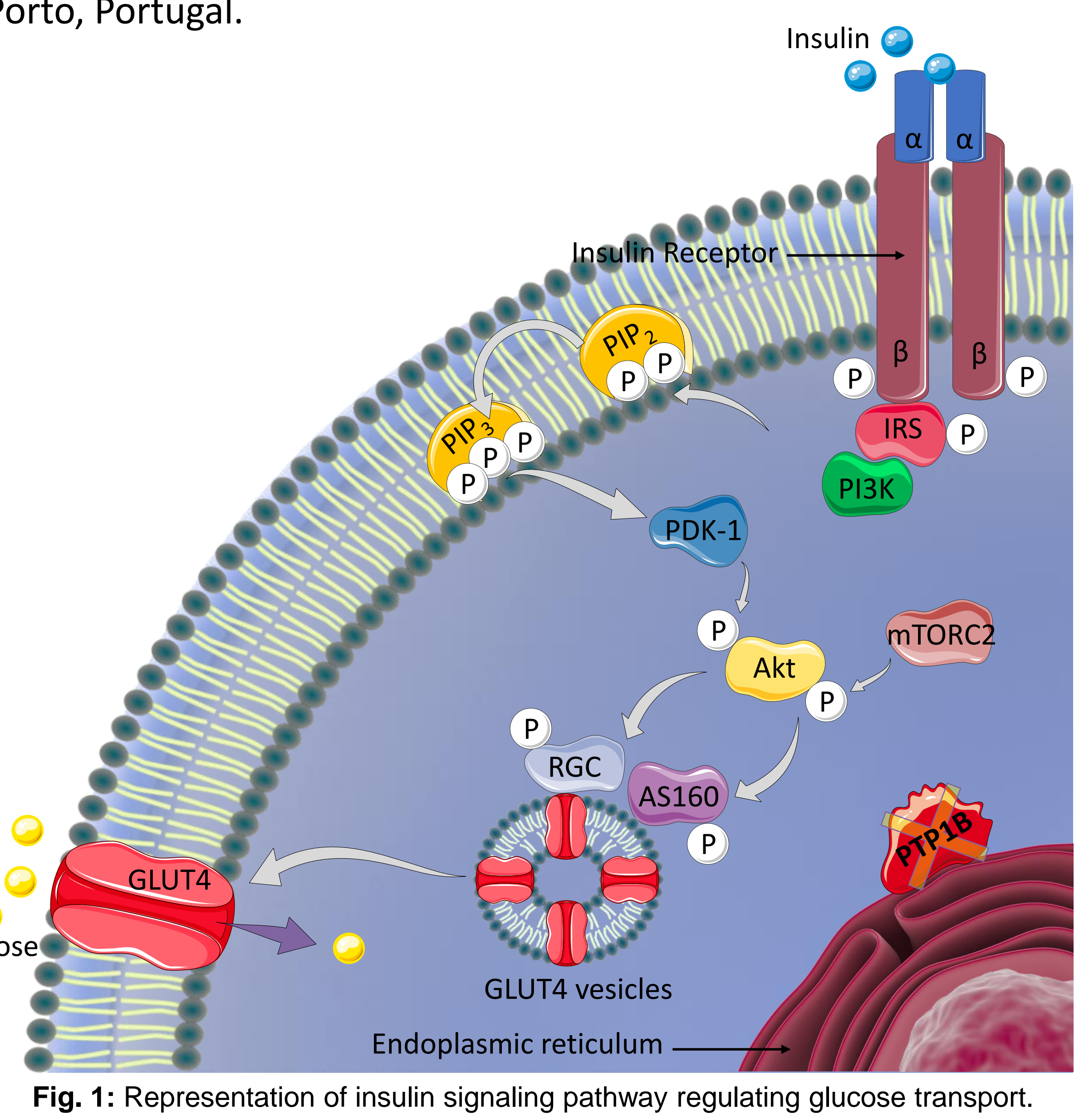

\section{Methods}

In vitro inhibitory assay for PTP1B activity

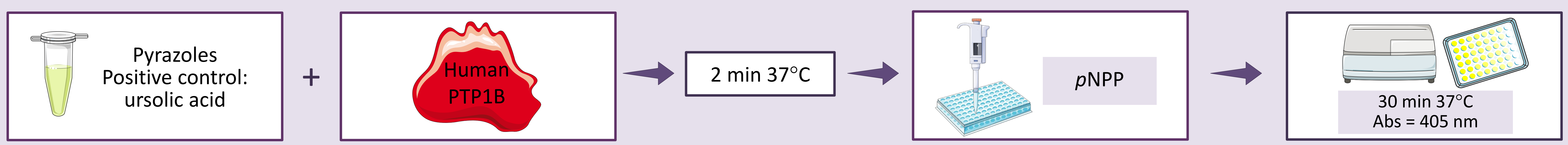

pNPP: $p$-nitrophenyl phosphate

\section{Results}

Table 1: Structures and in vitro PTP1B inhibition, expressed as \% inhibition (at the highest tested concentration indicated in superscript) and $\mathrm{I}_{50}$ values (mean \pm SEM) of the tested pyrazoles.

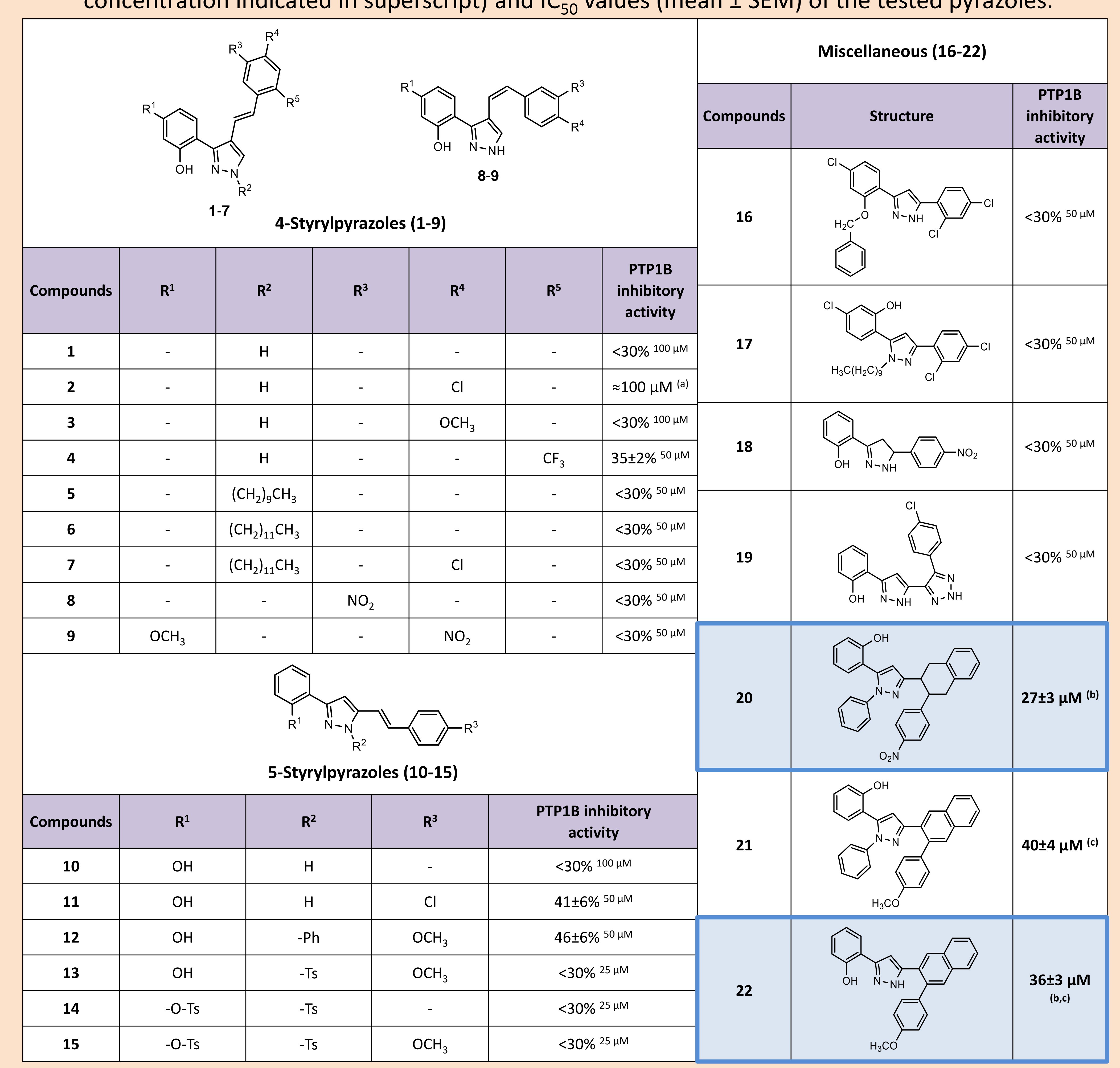

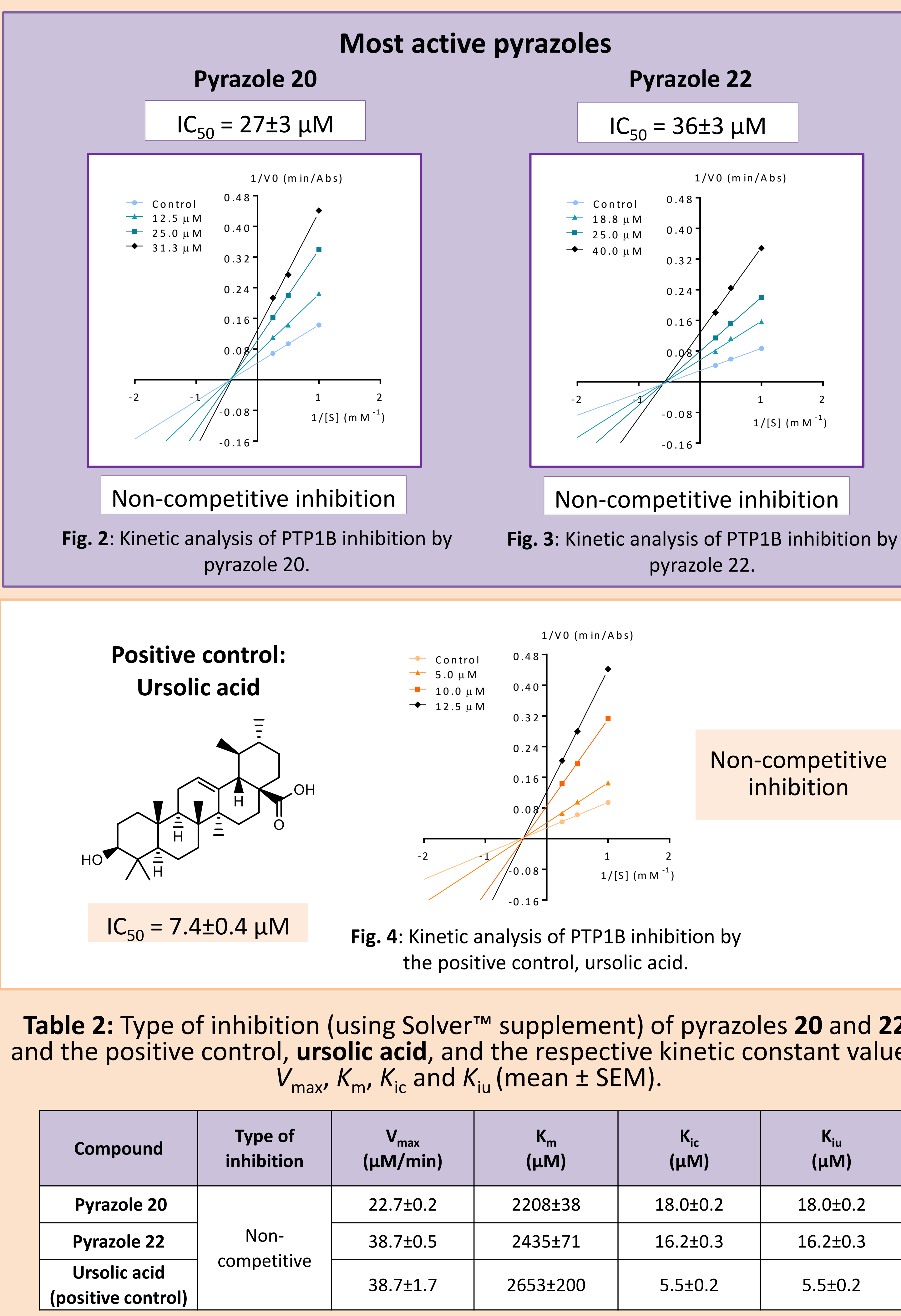

\section{Conclusions:}

$\checkmark$ It was possible to conclude that the type of substituents in the pyrazole moiety influence the inhibitory activity of the compounds. It was possible to conclude that the type of substituents in the pyrazole moiety influence the inhibitory activity of the compounds.
Pyrazole 20 and pyrazole 22 were the most promising compounds. The mechanism of inhibition was assessed using Lineweaver-Burk plots 